\title{
A Pilot Randomized Controlled Trial of Comparison between Extended Daily Hemodialysis and Continuous Veno-venous Hemodialysis in Patients of Acute Kidney Injury with Septic Shock
}

\author{
Shakti Bedanta Mishra, Afzal Azim¹, Narayan Prasad², Ratendra Kumar Singh¹, Banani Poddar', Mohan Gurjarr', Arvind Kumar Baronia ${ }^{1}$ \\ Department of Critical Care Medicine, IMS and SUM Hospital, Bhubaneswar, Odisha, Departments of ${ }^{1}$ Critical Care Medicine and ${ }^{2}$ Nephrology, \\ Sanjay Gandhi Postgraduate Institute of Medical Sciences, Lucknow, Uttar Pradesh, India
}

\section{Abstract}

\begin{abstract}
Aim of Study: Acute kidney injury (AKI) is common in patients of septic shock. There is sparse data comparing sustained low-efficiency dialysis (SLED) and continuous renal replacement therapy (CRRT) in patients with septic shock. Materials and Methods: This is a prospective randomized study in a 12-bedded medical intensive care unit. After clearance from institute's ethics committee and obtaining informed consent from the relatives, sixty adult patients with septic shock who were to undergo dialysis for AKI were included in the study. They were randomly assigned to SLED or CRRT group. Hemodynamic instability was defined as in terms of vasopressor dependency (VD). The worst value of VD during the dialysis session was taken into consideration. The primary objective was look at hemodynamic changes and secondarily into the efficacy. Results: The demographic data were comparable between the sixty patients randomized to thirty in each group. Delta VD and delta vasopressor index $(\Delta \mathrm{VI})$ were similar in SLED group compared to the CRRT group. CRRT group had better efficacy in terms of both equivalent renal urea clearance though fluid balance was not significantly better in CRRT group. Conclusion: SLED is a viable modality of renal replacement therapy in patients with septic shock as the hemodynamic effects are similar to CRRT.
\end{abstract}

Keywords: Acute kidney injury, dialysis, septic shock

\section{INTRODUCTION}

Acute kidney injury (AKI) develops in nearly $40 \%$ of the patients admitted in intensive care unit (ICU) with sepsis as the most common cause of development of AKI. ${ }^{[1]}$ These patients need dialysis if there is worsening in the renal parameters. In hemodynamically stable patients, the Kidney Disease Improving Global Outcome (KDIGO) guidelines recommend either intermittent or continuous renal replacement therapy (CRRT). ${ }^{[2]}$ CRRT is the preferred mode of renal replacement therapy (RRT) in patients with hemodynamic instability. The KDIGO guidelines of 2012 also suggest using CRRT in hemodynamically unstable patients.

Sustained low-efficiency dialysis (SLED) is a hybrid mode of dialysis with the advantages of CRRT and intermittent hemodialysis (IHD). SLED is a slower form of dialysis

\begin{tabular}{|l|l|}
\hline \multicolumn{3}{|c|}{ Access this article online } \\
\hline Quick Response Code: & Website: \\
\hline & www.ijccm.org \\
\hline
\end{tabular}

which maintains better hemodynamic stability as compared to $\mathrm{IHD},{ }^{[3]}$ and since it is intermittent, it also allows time for patient transport and procedures which is not possible with CRRT. SLED is gaining popularity worldwide due to its logistic advantages and apparent cost benefits even though the evidence is quite limited.

A recent meta-analysis compared SLED with CRRT in critically ill patients with AKI and revealed no difference in outcome between the two modalities. ${ }^{[4]}$ The authors found

Address for correspondence: Prof. Afzal Azim,

Department of Critical Care Medicine, Sanjay Gandhi Postgraduate Institute of Medical Sciences, Lucknow, Uttar Pradesh, India. E-mail: draazim2002@gmail.com

This is an open access article distributed under the terms of the Creative Commons Attribution-NonCommercial-ShareAlike 3.0 License, which allows others to remix, tweak, and build upon the work non-commercially, as long as the author is credited and the new creations are licensed under the identical terms.

For reprints contact: reprints@medknow.com

How to cite this article: Mishra SB, Azim A, Prasad N, Singh RK, Poddar B, Gurjar M, et al. A pilot randomized controlled trial of comparison between extended daily hemodialysis and continuous veno-venous hemodialysis in patients of acute kidney injury with septic shock. Indian J Crit Care Med 2017;21:262-7. 
mortality benefits in the observational trial in favor of SLED, but this finding could be attributed to possible allocation bias. In the same meta-analysis, there were three studies which have looked into the hemodynamic parameters, but they lacked any objective assessment of the hemodynamic perturbations during dialysis.

Septic AKI is a special subgroup associated with mortality rates up to $70 \%$ which is higher than other etiologies for AKIs. ${ }^{[5,6]}$ We conducted this study to compare the hemodynamic effects of CRRT versus SLED in patients with septic shock.

\section{Materials and Methods}

A prospective, randomized, single-center, two-group, parallel-group trial was carried out in a tertiary care hospital in North India. The study was conducted from July 1, 2014 to June 31, 2015. Institutional Review Board approval was obtained from the Research Ethics Committee of Sanjay Gandhi Post Graduate Institute of Medical Sciences, and the trial was registered in the Indian Clinical Trials Register (DRKS00004367). The study was conducted in accordance with the Declaration of Helsinki, October 2008 (49 ${ }^{\text {th }}$ General Assembly of the World Medical Association). All consent procedures followed local requirements, as approved by the ethics committee. The treating investigator informed the patient about the nature of the trial, its aims, and expected advantages, as well as possible risks. Written informed consent was obtained from eligible patients or by their legally authorized representatives. Deferred consent was used in emergencies, and a consultant physician independent of the investigational team gave authorization. Once the participant regained capacity or the legally authorized representative was available, the individual was asked to affirm or withdraw consent.

\section{Study population}

All adult patients, i.e., 18 years of age or older admitted to the ICU were screened for eligibility. The patients who were planned for RRT were screened for the presence of septic shock and AKI. Septic shock was defined according to the Surviving Sepsis Campaign guidelines of 2012. AKI was defined according to the KDIGO definition of 2012. The decision to initiate RRT, i.e., the timing of RRT was on the discretion of the treating physician. The exclusion criteria were vasopressor dependency (VD) of $<20$, age $>80$ years, metastatic cancer, decompensated cirrhosis, prior diagnosis of end-stage kidney disease, confirmed pregnancy, lack of commitment to medical treatment, and any session of dialysis received prior to admission. Source of sepsis was defined as the infection focus at the time of ICU admission.

\section{Randomization}

Eligible patients were randomly assigned to one of the two treatment groups by means of a computer-generated randomization software in 1:1 ratio. The patients allocated to one group underwent the modality of RRT till they recovered from shock. The modality of dialysis subsequently used was as per the discretion of the treating physician.

\section{Interventions}

\section{Sustained low-efficiency dialysis}

Fresenius 2008S dialysis machine and Fresenius AV600S $1.4 \mathrm{~m}^{2}$ (Fresenius Medical Care, Bad Hamburg, Germany) membranes were used in the study. The dialysis was to be done daily till the patient was off vasopressor. Planned dialysis duration was of $8 \mathrm{~h}$. Blood flow of $200 \mathrm{ml} / \mathrm{min}$ and dialysate flow of $250 \mathrm{ml} / \mathrm{min}$ were used. Dialysate fluids were manufactured from tap water at the bedside.

\section{Continuous renal replacement therapy}

Fresenius multiFiltrate Kit $3 \mathrm{CVVH}$ dialysis machine and Fresenius Ultraflux ${ }^{\circledR}$ AV 600 S (Fresenius Medical Care, Bad Hamburg, Germany) membranes were used in the study. CRRT was done for duration of $72 \mathrm{~h}$ and reviewed for further need. Blood flow of $150 \mathrm{ml} / \mathrm{min}$ and dialysate rate of $30 \mathrm{ml} / \mathrm{kg} / \mathrm{h}$ were used.

Anticoagulation during dialysis and the ultrafiltration rate were decided by the treating clinician. Serum values for small solutes (urea and creatinine), electrolytes, $\mathrm{pH}$, bicarbonate, and base excess were assessed before the start of each mode of RRT, at $12 \mathrm{~h}$, and daily at $0500 \mathrm{~h}$ thereafter. Vasopressors were used to target a mean arterial pressure (MAP) of $\geq 65 \mathrm{mmHg}$.

\section{Study outcomes}

The primary outcome measure was hemodynamic stability by delta VD $(\Delta V D)$. Secondary outcome was efficacy by fluid balance in interdialytic period and equivalent renal urea clearance (EKRjc).

Hemodynamics was described using vasopressor index (VI) and VD. The VI was calculated by the following formula (dopamine dose $\times 1)+($ dobutamine dose $\times 1)+($ adrenaline dose $\times 100)$ $+($ noradrenaline dose $\times 100)+($ phenylephrine dose $\times 100)$ $+($ vasopressin dose $\times 10) .{ }^{[7-9]}$ All doses are in $\mu \mathrm{g} / \mathrm{kg} / \mathrm{min}$ except that of vasopressin which is in units/h. We modified the index to include vasopressin as it is the most commonly used vasopressor after noradrenaline in septic shock. $\mathrm{VD}^{[10]}$ was calculated by the following formula VI/MAP $\times 100$. This was done to negate the effect of MAP achieved. The delta VI $(\Delta \mathrm{VI})$ and $\Delta \mathrm{VD}$ were calculated by the difference between the predialysis values from the worst level recorded during the dialysis session. The comparison of hemodynamics between the two groups was done by analyzing the worst $\Delta \mathrm{VD}$ value of each patient.

Daily fluid balance was defined as the total fluid intake from all sources (intravenous fluids and blood products, enteral and parenteral nutrition, and medications) minus the output from all sources (urine, ultrafiltrate, and output from drains). Since the gastrointestinal losses (stool volume) were not quantified, they were not included in calculations. We calculated mean daily fluid balance for the entire dialysis period for analysis. Casino and Marshall[11] in virtual patients using a variable volume double pool urea kinetic model showed that EKRjc 
was an effective method to calculate the dose of dialysis in both intermittent and continuous forms of dialysis. The dosage was calculated using the formula in a spreadsheet computer application. The mean daily fluid balance and dosage was calculated till the period of vasopressor requirement. The cost analysis was based on cost for the dialysis for each patient during the study period recorded in USD.

\section{Statistical analysis}

Prior to this trial, we had done an observational study of 124 patients with septic shock undergoing SLED in our ICU. ${ }^{[9]}$ In those patients, we found that those patients whose VD is more than 25 prior to initiation of dialysis had more instability during SLED. We hypothesized that CRRT will be able to reduce the hemodynamic instability during dialysis in this patient population. In the study, we found mean $\Delta \mathrm{VD}$ to be 19 with a standard deviation (SD) of 19 in this group of patients. We estimated that CRRT would be able to reduce the mean $\triangle \mathrm{VD}$ to 5 considering that it has better hemodynamic tolerability. Hence, based on this, to have $80 \%$ power with $5 \% \alpha$ error, we estimated the required sample size to be 29 in each group with 1:1 allocation.

All analyses were performed according to the intention-to-treat principle. Comparison of qualitative data was performed using Chi-square analysis or by Fisher's exact test. For quantitative analysis, differences between means were identified using independent $t$-test for independent variables. Statistical significance was accepted at $P<0.05$. All statistical analyses were performed using Statistical Package for the Social Sciences software version 17 (IBM SPSS Statistics, Version 17.0. Armonk, NY: IBM Corp).

\section{RESULTS}

The study was conducted in the period from July 1, 2014 to June 31, 2015. Out of a total of 243 patients admitted to our ICU, sixty patients were included in the trial. Thirty patients were randomized to each group. The consort diagram is shown in Figure 1.

\section{Demographics}

The age distribution and admission APACHE II and SOFA scores were similar between the groups [Table 1]. There were nine female patients each in both the groups. Stage 3 was the most common staging of AKI with which patients were admitted to the ICU. The most common source of sepsis was pneumonia followed by intra-abdominal infection. The baseline laboratory parameters were comparable. The renal and acid-base parameters were comparable prior to the study inclusion between the two groups [Table 2].

\section{Dialysis sessions}

Ninety-eight sessions of SLED were analyzed. The median days per patient were 3.5 days. The mean duration of dialysis was $7.4 \pm 1.1 \mathrm{~h}($ mean $\pm \mathrm{SD})$. The mean blood flow in these sessions was $173.4 \pm 23.5 \mathrm{ml} / \mathrm{min}$. The mean dialysate flow was $220.3 \pm 38.1 \mathrm{ml} / \mathrm{h}$. The mean ultrafiltrate per session was $1633.07 \pm 733.2 \mathrm{ml}$.
Totally 42 sessions of CRRT were analyzed. The median days per patient were 3 days. The mean duration of dialysis was $68.3 \pm 10.3 \mathrm{~h}$ (mean $\pm \mathrm{SD}$ ). The mean blood flow in these sessions was $150 \pm 33 \mathrm{ml} / \mathrm{min}$. The mean dialysate flow was $899 \pm 167.2 \mathrm{ml} / \mathrm{h}$. The mean ultrafiltrate per session was $88.2 \pm 22.1 \mathrm{ml} / \mathrm{h}$.

\section{Hemodynamics}

The predialysis MAP, VI, and VD were similar in both the groups [Table 3]. The intradialysis hypotension which was

\begin{tabular}{|c|c|c|c|}
\hline Variables & SLED $(n=30)$ & CRRT $(n=30)$ & $P$ \\
\hline Age (years) & $47.8 \pm 16$ & $49 \pm 16$ & 0.81 \\
\hline Female, $n(\%)$ & $9(30)$ & $9(30)$ & $>0.99$ \\
\hline \multicolumn{4}{|l|}{ Severity scoring } \\
\hline APACHE II & $24.6 \pm 6.5$ & $25.6 \pm 6.6$ & 0.90 \\
\hline SOFA & $12.4 \pm 4.1$ & $13.3 \pm 3.4$ & 0.55 \\
\hline \multicolumn{4}{|l|}{ AKI stage, $n(\%)$} \\
\hline 1 & $3(10)$ & $4(13)$ & 0.85 \\
\hline 2 & $6(20)$ & $7(23)$ & \\
\hline 3 & $21(70)$ & $19(64)$ & \\
\hline \multicolumn{4}{|l|}{ Comorbidities, $n(\%)$} \\
\hline Diabetes mellitus & $17(57)$ & $12(40)$ & 0.30 \\
\hline Hypertension & $13(43)$ & $12(40)$ & 0.79 \\
\hline COPD & $4(13)$ & $7(23)$ & 0.31 \\
\hline Coronary artery disease & $1(3)$ & $1(3)$ & $>0.99$ \\
\hline \multicolumn{4}{|l|}{ Source of sepsis, $n(\%)$} \\
\hline Respiratory & $15(50)$ & $15(50)$ & 0.72 \\
\hline Intra-abdominal & $11(37)$ & $10(33)$ & \\
\hline Hematological & $1(3)$ & $2(7)$ & \\
\hline Others & $2(7)$ & $3(10)$ & \\
\hline Unknown & $1(3)$ & 0 & \\
\hline \multicolumn{4}{|l|}{ Laboratory parameters } \\
\hline Hemoglobin (g/dL) & $9.4 \pm 3$ & $9.3 \pm 2.4$ & 0.98 \\
\hline $\mathrm{TLC} \times 10^{3} / \mu \mathrm{L}$ & $19 \pm 12$ & $17 \pm 11$ & 0.21 \\
\hline Platelet $\times 10^{9} / \mathrm{L}$ & $156 \pm 120$ & $139 \pm 86$ & 0.14 \\
\hline aPTT (s) & $35 \pm 8$ & $38 \pm 10$ & 0.69 \\
\hline Prothrombin time (s) & $1.9 \pm 1.1$ & $2.3 \pm 1.7$ & 0.25 \\
\hline Pro-calcitonin (ng/mL) & $23 \pm 10$ & $20 \pm 8$ & 0.10 \\
\hline
\end{tabular}

All data are in median and IQR unless specified. SLED: Sustained low-efficiency dialysis; CRRT: Continuous renal replacement therapy; APACHE: Acute Physiology And Chronic Health Evaluation;

SOFA: Sequential organ failure assessment; COPD: Chronic obstructive pulmonary disease; TLC: Total leukocyte count; aPTT: Activated partial thromboplastin time; AKI: Acute kidney injury; IQR: Interquartile range

\begin{tabular}{lccc}
\hline \multicolumn{4}{l}{ Table 2: Predialysis variables } \\
\hline Variables & SLED $(\boldsymbol{n = 3 0})$ & CRRT $(\boldsymbol{n = 3 0})$ & $\boldsymbol{P}$ \\
\hline Creatinine $(\mathrm{mg} / \mathrm{dL})$ & $3.7 \pm 1.8$ & $3.9 \pm 1.9$ & 0.36 \\
Blood urea nitrogen $(\mathrm{mg} / \mathrm{dL})$ & $66 \pm 44$ & $62 \pm 36$ & 0.26 \\
pH & $7.28 \pm 0.13$ & $7.25 \pm 0.10$ & 0.47 \\
Bicarbonate $(\mathrm{mEq} / \mathrm{L})$ & $17 \pm 4.5$ & $16 \pm 3.5$ & 0.23 \\
Base deficit $(\mathrm{mEq} / \mathrm{L})$ & $8.5 \pm 6.2$ & $9.6 \pm 4.6$ & 0.36 \\
Potassium $(\mathrm{mEq} / \mathrm{L})$ & $4.7 \pm 1.7$ & $4 \pm 2$ & 0.27 \\
\hline
\end{tabular}

SLED: Sustained low-efficiency dialysis; CRRT: Continuous renal replacement therapy 


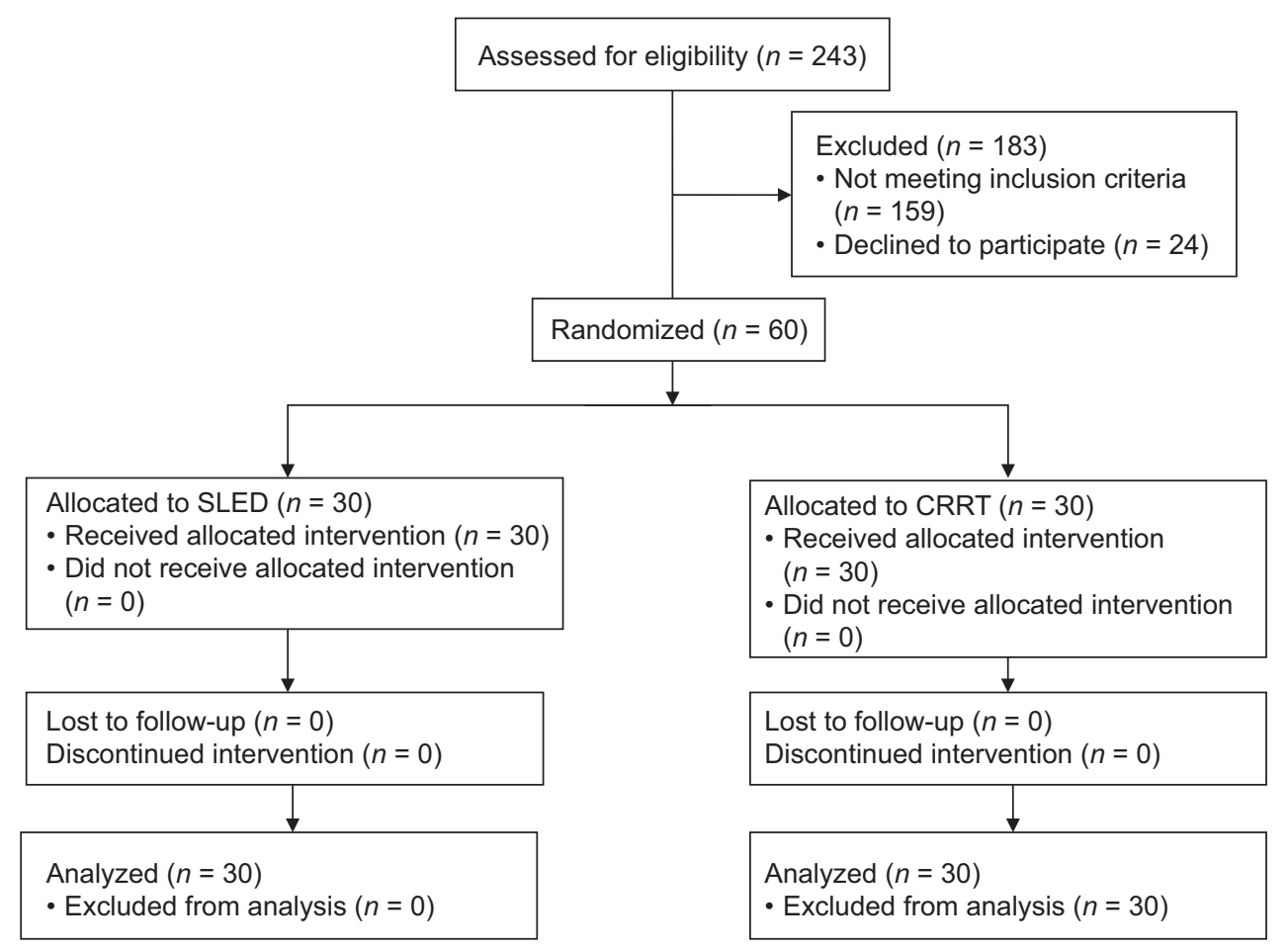

Figure 1: Consort flow diagram

measured in terms of $\triangle \mathrm{VI}$ in SLED group was similar than that of CRRT group (SLED mean $26 \pm 33$ vs. CRRT mean $25 \pm 44 ; P=0.42)$. $\triangle$ VD was also similar in SLED group compared to the CRRT group (SLED mean $39 \pm 40$ vs. CRRT mean $42 \pm 51 ; P=0.39$ ) [Table 3].

\section{Efficacy}

The efficacy of the sessions was calculated by EKRjc [Table 3]. The mean EKRjc was significantly higher $(P=0.04)$ in CRRT group $(33.9 \pm 15)$ compared to SLED group $(29.2 \pm 10)$. CRRT provided significantly $(P=0.10)$ lower fluid balance $(0.68 \pm 0.20 \mathrm{~L} /$ day $)$ when compared to SLED which provided $0.79 \pm 0.24 \mathrm{~L} /$ day of balance. The cost of therapy in SLED was $226.15 \pm 177.4$ USD per person which was significantly less than that of CRRT group which had an average cost of $722.0 \pm 398.1$ USD $(P<0.01)$.

\section{Discussion}

Our study aimed to investigate whether SLED is both hemodynamically tolerable and efficacious in patients with septic shock in comparison to CRRT. The definition of hemodynamic tolerability in literature is ambiguous. ${ }^{[12]}$ Several definitions have been used in studies comparing IHD or SLED with CRRT. Defining hemodynamic instability by a decrease in MAP is not informative when the practice is to achieve a target MAP by titrating the dose of vasopressor infusion rates. The recent meta-analysis comparing SLED and CRRT also commented that no relevant data could be extracted about hemodynamic management of septic shock when patients were already on vasopressors ${ }^{[4]}$ and the MAPs are maintained.

\begin{tabular}{lccc}
\hline \multicolumn{4}{l}{ Table 3: Hemodynamics and efficacy } \\
\hline & SLED $(\boldsymbol{n = 3 0})$ & CRRT $(\boldsymbol{n = 3 0})$ & $\boldsymbol{P}$ \\
\hline VI (predialysis) & $59 \pm 33$ & $66 \pm 42$ & 0.15 \\
VD (/mmHg) (predialysis) & $78 \pm 53$ & $78 \pm 54$ & 0.81 \\
MAP (mmHg) (predialysis) & $78 \pm 11$ & $78 \pm 12$ & 0.62 \\
VI & $86 \pm 52$ & $92 \pm 51$ & 0.92 \\
VD (/mmHg) & $129 \pm 99$ & $121 \pm 61$ & 0.10 \\
Heart rate (/min) & $98 \pm 12$ & $96 \pm 13$ & 0.24 \\
MAP (mmHg) (intradialysis) & $82 \pm 11$ & $81 \pm 10$ & 0.49 \\
$\Delta$ VI & $26 \pm 33$ & $25 \pm 44$ & 0.42 \\
$\Delta$ VD & $39 \pm 40$ & $42 \pm 51$ & 0.39 \\
EKRjc & $29.2 \pm 10$ & $33.9 \pm 15$ & 0.04 \\
Fluid balance (1/24 h) & $0.79 \pm 0.24$ & $0.68 \pm 0.20$ & 0.10 \\
Cost (USD) & 226.15 \pm 177.4 & $722.0 \pm 398.1$ & $<0.01$ \\
\hline All data are in median and IQR unless specified. SLED: Sustained \\
low-efficiency dialysis; CRRT: Continuous renal replacement therapy; \\
$\Delta$ VI: Delta vasopressor index; $\Delta$ VD: Delta vasopressor dependency; \\
EKRjc: Equivalent renal urea clearance; MAP: Mean arterial pressure; \\
IQR: Interquartile range
\end{tabular}

In this context, the cutoff vasopressor dose beyond which it is raised may more suitably define hemodynamic instability. Hence, we used a more objective and comparable concept of VI and VD in our study. Both these concepts have been used previously. Hemodynamic instability described by VD nullifies the effect of MAP, which may vary from patient to patient and in specific patient populations.

This is the first study to the best of our knowledge that compares SLED and CRRT in patients with septic shock. Kielstein et al..$^{[13]}$ compared SLED and CRRT in critically 
ill patients. This randomized study in critically ill patients examined hemodynamic stability in terms of variability in heart rate, MAP, cardiac output, and systemic vascular resistance. Correction of metabolic acidosis during the course of dialysis was their end point. No difference was observed between SLED and CRRT. Their mean noradrenaline dose of $0.47 \mu \mathrm{g} / \mathrm{kg} / \mathrm{min}$ was higher compared to our study wherein it was only $0.19 \mu \mathrm{g} / \mathrm{kg} / \mathrm{min}$. Only directional trends of vasopressors were reported rather than the exact quantification as attempted in our study. Fieghen et al. ${ }^{[14]}$ examined hemodynamic stability in a mixed group of critically ill patients. Hemodynamic instability was defined as reduction in MAP $>20 \%$ or escalation in vasopressor dose. These investigators in their comparative nonrandomized study inferred that SLED is comparable to CRRT in critically ill patients. However, only $70 \%$ of their patients were in shock and even among them not all were in septic shock. They also did not quantify the mean dose of noradrenaline requirement. Although their patients on SLED had higher episodes of hemodynamic instability ( $38.5 \%$ vs. $18.5 \%$ in CRRT), the requirements for vasopressor escalation were more in CRRT (39.5\% vs. $25.6 \%$ in SLED). Baldwin et al. ${ }^{[15]}$ in a comparative (SLED vs. CRRT) randomized controlled trial of 16 patients concluded that SLED was more effective in fluid removal, though with lower MAPs compared to CRRT in critically ill patients.

We have described hemodynamics in terms of VI and VD. The population that we included had VD more than 20 which was an inclusion criterion prior to randomization. The hemodynamics in terms of VI, VD, and MAP was similar in both the groups prior to initiation of dialysis. The $\Delta \mathrm{VI}$ and $\Delta \mathrm{VD}$ were similar in both the groups. We had presumed that CRRT considering its theoretical advantage over SLED would result in greater reduction of $\triangle \mathrm{VD}$. However, both the groups were comparable in this context. This is a major finding in our study as the main utility of CRRT lies with hemodynamically unstable patients.

Efficacy of a dialysis session is mainly denoted by its dosing, i.e., by the Kt/v. ${ }^{[16]}$ The dosing of CRRT and SLED cannot be compared by this method as Kt/v cannot be done for continuous dialysis. The EKRjc was utilized for this purpose. Casino and Marshall ${ }^{[1]}$ have shown that this is a reliable method for measuring the dosing in both intermittent and continuous methods of dialysis. The results show that CRRT was better in terms of dosing compared to SLED. The fluid balance expressed in balance per $24 \mathrm{~h}$ was also better in CRRT though not statistically significant. Baldwin et al. ${ }^{[15]}$ in a randomized controlled trial of 16 patients comparing SLED with CRRT concluded that SLED was more effective in fluid removal, despite lower MAPs in critically ill patients. No significant difference was observed between groups for heart rate, central venous pressure, and noradrenaline dose. Our study shows that in CRRT the EKRjc was significantly higher in comparison to SLED which signifies that the solute removal was better with CRRT.

Fluid removal is an important aspect of management in hemodialysis of hemodynamically unstable patients. The ultra-filtration achieved during the dialysis sessions could not be compared as the duration of dialysis was different in the two modalities. Daily fluid balance has been shown to be an effective way of noting the fluid management in critically ill patients and this has been shown to be of value in patients who undergo dialysis. Silversides et al.$^{[17]}$ have demonstrated that fluid balance and interdialysis hypotension during dialysis were predictors of mortality in AKI. Our study shows that CRRT is better than SLED in terms of maintenance of fluid balance. The fluid removal was not significantly different between the modalities though it was lower in CRRT. This could be especially important in patients of raised intracranial pressure and ARDS in whom tight control of fluid balance is paramount.

Our study limitations include being a single-center study with a small sample size. We did not compare outcome parameters such as length of ICU stay, ventilator duration, and recovery of renal function in follow-up. Our strength is the study design and the objective assessment of hemodynamics which has not been done before in patients with septic shock.

\section{Conclusion}

Our study showed similar hemodynamic effects of CRRT and SLED in patients with septic shock. SLED was cost-effective compared to CRRT. It provides a basis for future larger studies with better objective assessment of hemodynamics in patients with septic shock.

\section{Financial support and sponsorship}

Nil.

\section{Conflicts of interest}

There are no conflicts of interest.

\section{RefERences}

1. Liaño F, Junco E, Pascual J, Madero R, Verde E. The spectrum of acute renal failure in the Intensive Care Unit compared with that seen in other settings. The Madrid Acute Renal Failure Study Group. Kidney Int Suppl 1998;66:S16-24.

2. Kidney Disease: Improving Global Outcomes (KDIGO) Acute Kidney Injury Work Group. KDIGO clinical practice guideline for acute kidney injury. Kidney Int Suppl 2012;1:1-138.

3. Neves JB, Rodrigues FB, Castelão M, Costa J, Lopes JA. Extended daily dialysis versus intermittent hemodialysis for acute kidney injury: A systematic review. J Crit Care 2016;33:271-3.

4. Zhang L, Yang J, Eastwood GM, Zhu G, Tanaka A, Bellomo R. Extended daily dialysis versus continuous renal replacement therapy for acute kidney injury: A meta-analysis. Am J Kidney Dis 2015;66:322-30.

5. Ostermann M, Chang RW. Acute kidney injury in the Intensive Care Unit according to RIFLE. Crit Care Med 2007;35:1837-43.

6. Uchino S, Kellum JA, Bellomo R, Doig GS, Morimatsu H, Morgera S, et al. Acute renal failure in critically ill patients: A multinational, multicenter study. JAMA 2005;294:813-8.

7. Wernovsky G, Wypij D, Jonas RA, Mayer JE Jr., Hanley FL, Hickey PR, et al. Postoperative course and hemodynamic profile after the arterial switch operation in neonates and infants. A comparison of low-flow cardiopulmonary bypass and circulatory arrest. Circulation 1995;92:2226-35.

8. Zuppa AF, Nadkarni V, Davis L, Adamson PC, Helfaer MA, Elliott MR, et al. The effect of a thyroid hormone infusion on vasopressor support in critically ill children with cessation of neurologic function. Crit Care 
Med 2004;32:2318-22.

9. Mishra SB, Singh RK, Baronia AK, Poddar B, Azim A, Gurjar M. Sustained low-efficiency dialysis in septic shock: Hemodynamic tolerability and efficacy. Indian J Crit Care Med 2016;20:701-7.

10. Cruz DN, Antonelli M, Fumagalli R, Foltran F, Brienza N, Donati A, et al. Early use of polymyxin B hemoperfusion in abdominal septic shock: The EUPHAS randomized controlled trial. JAMA 2009;301:2445-52.

11. Casino FG, Marshall MR. Simple and accurate quantification of dialysis in acute renal failure patients during either urea non-steady state or treatment with irregular or continuous schedules. Nephrol Dial Transplant 2004;19:1454-66.

12. Rabindranath K, Adams J, Macleod AM, Muirhead N. Intermittent versus continuous renal replacement therapy for acute renal failure in adults. Cochrane Database Syst Rev 2007;3:CD003773.

13. Kielstein JT, Schiffer M, Hafer C. Back to the future: Extended dialysis for treatment of acute kidney injury in the Intensive Care Unit. J Nephrol
2010;23:494-501.

14. Fieghen HE, Friedrich JO, Burns KE, Nisenbaum R, Adhikari NK, Hladunewich MA, et al. The hemodynamic tolerability and feasibility of sustained low efficiency dialysis in the management of critically ill patients with acute kidney injury. BMC Nephrol 2010;11:32.

15. Baldwin I, Bellomo R, Naka T, Koch B, Fealy N. A pilot randomized controlled comparison of extended daily dialysis with filtration and continuous veno-venous hemofiltration: Fluid removal and hemodynamics. Int J Artif Organs 2007;30:1083-9.

16. Marshall MR, Golper TA, Shaver MJ, Alam MG, Chatoth DK. Urea kinetics during sustained low-efficiency dialysis in critically ill patients requiring renal replacement therapy. Am J Kidney Dis 2002;39:556-70.

17. Silversides JA, Hladunewich MA, Lapinsky SE, Pinto R, Kuint R, Wald R, et al. Fluid balance, intradialytic hypotension, and outcomes in critically ill patients undergoing renal replacement therapy: A cohort study. Crit Care 2014;18:624. 